\title{
Evolution of species complementarity in response to drought in a grassland biodiversity experiment
}

Yuxin Chen ( $\nabla$ yuxin.chen@xmu.edu.cn )

Xiamen University https://orcid.org/0000-0003-0658-7562

Anja Vogel

Leipzig University

Cameron Wagg

University of Zürich https://orcid.org/0000-0002-9738-6901

Tianyang $\mathrm{Xu}$

University of Zürich

Maitane Iturrate-Garcia

University of Zurich

Michael Scherer-Lorenzen

University of Freiburg https://orcid.org/0000-0001-9566-590X

Alexandra Weigelt

University of Leipzig https://orcid.org/0000-0001-6242-603X

Nico Eisenhauer

German Center for Integrative Biodiversity Research https://orcid.org/0000-0002-0371-6720

Bernhard Schmid

University of Zurich

Article

Keywords:

Posted Date: May 17th, 2021

DOl: https://doi.org/10.21203/rs.3.rs-530107/v1

License: (c) (1) This work is licensed under a Creative Commons Attribution 4.0 International License.

Read Full License 
1 Title: Evolution of species complementarity in response to drought in a grassland

biodiversity experiment

\section{Short title: Drought-induced selection for species complementarity}

\section{Authors}

Yuxin Chen ${ }^{1,2,3, *}$, Anja Vogel ${ }^{4,5,6}$, Cameron Wagg ${ }^{2,7}$, Tianyang $\mathrm{Xu}^{2}$, Maitane Iturrate Garcia $^{2,8}$, Michael Scherer-Lorenzen ${ }^{9}$, Alexandra Weigelt ${ }^{4,10}$, Nico Eisenhauer ${ }^{4,5}$, Bernhard Schmid ${ }^{11,12, *}$

\section{Affiliations:}

${ }^{1}$ Key Laboratory of the Ministry of Education for Coastal and Wetland Ecosystems, College of the Environment \& Ecology, Xiamen University, 361102 Xiamen, China.

${ }^{2}$ Department of Evolutionary Biology and Environmental Studies, University of Zürich, Winterthurerstrasse 190, 8057 Zürich, Switzerland.

${ }^{3}$ School of Life Sciences/State Key Laboratory of Biocontrol, Sun Yat-sen University, 510275 Guangzhou, China.

${ }^{4}$ German Centre for Integrative Biodiversity Research (iDiv) Halle-Jena-Leipzig, Puschstraße 4, 04103 Leipzig, Germany.

${ }^{5}$ Institute of Biology, Leipzig University, Puschstraße 4, 04103 Leipzig, Germany

${ }^{6}$ Institute of Ecology and Evolution, Friedrich Schiller University Jena, 07743 Jena, Germany.

${ }^{7}$ Fredericton Research and Development Center, Agriculture and Agri-Food Canada, 850 Lincoln Road, Fredericton, New Brunswick, E3B 4Z7, Canada.

${ }^{8}$ Department of Chemical and Biological Metrology, Federal Institute of Metrology METAS, Lindenweg 50, 3003 Bern-Wabern, Switzerland 
$25{ }^{9}$ Geobotany, Faculty of Biology, University of Freiburg, 79106 Freiburg, Germany.

$26{ }^{10}$ Institute of Biology, Leipzig University, Johannisallee 21-23, 04103 Leipzig,

27 Germany

$28{ }^{11}$ Department of Geography, Remote Sensing Laboratories, University of Zürich,

29 Winterthurerstrasse 190, 8057 Zürich, Switzerland.

$30{ }^{12}$ Institute of Ecology, College of Urban and Environmental Sciences, Peking

31 University, 100871 Beijing, China.

32 *Correspondence: yuxin.chen@xmu.edu.cn and bernhard.schmid@uzh.ch

33 Type of article: Article

34 


\section{Abstract}

Growing threats from extreme climatic events and biodiversity loss have raised concerns about their interactive consequences for ecosystem functioning. Evidence suggests that biodiversity is crucial to buffer ecosystem functioning facing climatic extremes. However, whether evolutionary processes in species mixtures underpin such biodiversity-dependent stabilizing effects remains elusive. We tested this hypothesis by exposing experimental mixtures of grassland species to eight recurrent summer droughts vs. control in the field. Seed offspring of 12 species were subsequently grown individually, in monocultures or in 2-species mixtures and subjected to a novel drought event in the glasshouse. Comparing mixtures with monocultures, drought-selected plants showed greater between-species complementarity than ambient-selected plants when recovering from the drought event, which led to greater biodiversity effects on community productivity and better recovery of drought-selected mixtures after the drought. These findings suggest biodiversity can buffer the impacts of extreme climatic events through evolution of species complementarity.

\section{Main text}

Extreme climatic events such as droughts are predicted to be more frequent in the future $^{1,2}$, with potentially negative effects on the functioning of ecosystems and the provision of ecosystem services for human well-being ${ }^{3-7}$. Ecosystems may adjust to climatic extremes by changes in species abundances ${ }^{8-10}$ or evolutionary responses of populations ${ }^{11-19}$. Both experimental and observational studies have shown that higher biodiversity buffers temporal variation in ecosystem functioning against extreme climatic events ${ }^{20-24}$. Stabilizing effects can arise from various ecological mechanisms, 
such as portfolio effects due to the scaling relationship between the mean and the variance ${ }^{25}$, complementarity among species in responses to environmental fluctuations ${ }^{26}$, and compensatory recovery in which increased recovery after extreme climatic events compensates for the event-driven loss of ecosystem functioning ${ }^{23}$. However, large gaps remain in our empirical understanding of whether and how evolution may underpin such stabilizing effects of biodiversity on ecosystem functioning in the face of extreme climatic events. Rapid evolutionary change under such climatic perturbations may arise from a filtering of pre-existing genetic variation via differential demographic processes ${ }^{27,28}$.

Extreme climatic events may select for specific plant traits with the consequence of higher tolerance under future climatic extreme events or faster recovery after such events. For example, drought may select for higher tolerance of water deficits (e.g., shorter height, greater leaf mass per area, higher turgor loss point, or higher root-shoot biomass ratio) ${ }^{29-32}$, which may reduce plant mortality rates and increase community resistance to drought. Alternatively, drought may select for faster recovery after drought ${ }^{13,17}$. Nutrient availability such as soil nitrogen may increase after drought and favor the growth of acquisitive individuals (e.g., with lower leaf mass per area, or higher leaf and root nitrogen content) that survived post drought ${ }^{32-34}$. As a consequence, community productivity may also be increased after drought ${ }^{23}$. Extreme climatic events may also induce the evolution of species interactions within communities in complex ways ${ }^{12,35-37}$, affecting biodiversity effects on ecosystem functioning. First, selection for specific traits may change the average dissimilarity and niche difference between species within communities (Supplementary Fig. 1). For example, drought may exclude intolerant genotypes from intolerant species, which may increase the niche overlap and competition intensity 
between intolerant and tolerant species ${ }^{38}$ (Supplementary Fig. 1a). Alternatively, drought may exclude intolerant genotypes from both intolerant and tolerant species, which may reduce the niche overlap and competition intensity between the two species (Supplementary Fig. 1b). Second, trait expression in one species may influence trait expression in another interacting species, leading to heritable changes in species interactions ${ }^{37}$. That is, selection may occur at the community level and not be inferred from the sum or average of trait variation of individual species ${ }^{37}$. Selection for reduced species competition and niche overlap could strengthen positive biodiversity effects ${ }^{39,40}$.

Experiments have shown that evolved species interactions strengthened the positive biodiversity effects on productivity in microbial communities ${ }^{41,42}$ and in grassland communities under benign environments ${ }^{43-45}$. But whether and how extreme climatic events will lead to evolutionary changes in species interactions, and further change the biodiversity effects in plant communities remains elusive.

In the present study, we assessed how an 8-year selection treatment of summer droughts affected plant diversity effects on ecosystem functioning and species interactions before, during and after a novel drought event. We exposed experimental mixtures of grassland species to eight recurrent summer droughts vs. control in a long-term biodiversity experiment in the field (the Jena Experiment ${ }^{23,46,47}$ ). Seed offspring of 12 species were subsequently grown individually, in monocultures or in 2-species mixtures and subjected to a novel drought event in the glasshouse (Fig. 1; Supplementary Table 1). In the glasshouse experiment, both monocultures and mixtures contained four individuals per pot. We harvested aboveground biomass (a proxy for productivity) of every pot three times: (1) after a first phase of three months with regular watering (ambient conditions, "before drought"); (2) after a second phase 
of two weeks without watering (drought conditions, "during drought") and (3) after a third phase of seven weeks with regular watering (ambient conditions for recovery, "after drought").

We used the harvested aboveground biomass in four sets of analyses. First, we assessed how drought selection affected biodiversity effects on productivity before, during and after the drought event in the glasshouse. We used the biomass difference between 2 -species mixtures vs. monocultures to calculate the net biodiversity effects, which we further partitioned into complementarity effects (indicating niche differentiation or facilitation) and sampling effects (indicating a disproportionate contribution to community productivity of one or the two species; which is also referred as "selection effect" elsewhere, a term we did not use in this study to avoid confusion ${ }^{48}$. Second, we assessed the effects of drought selection on resistance (biomass ratio during vs. before the drought), recovery (biomass ratio after vs. during the drought) and resilience (biomass ratio after vs. before the drought) of productivity 19,20. Third, we tested whether altered plant interactions drove the above differences between the two selection treatments. We calculated the intensities of neighbor interactions for mixtures (i.e., heterospecific interactions) and monocultures (i.e., conspecific interactions) separately, and then calculated their difference as a proxy for niche difference. Fourth, we assessed how drought selection influenced trait values on plants in pots with one individual and trait dissimilarities between interacting species in mixtures.

The objectives of this study were to (1), assess how drought selection affected biodiversity effects on productivity and stability over different phases of an experimental drought event; and to (2), investigate the roles of species interactions in driving the evolutionary changes in biodiversity effects on productivity and stability. 


\section{RESULTS}

137

\section{Effects of drought selection on biodiversity effects on productivity}

We found that the net biodiversity effects for different species pairs were higher when plants had been selected under repeated summer droughts in the field, but this only became visible when their productivity was assessed after the drought event in the glasshouse (Figs. $2 \& 3$, Supplementary Tables $2 \& 3$ ). The positive net biodiversity effects in mixtures of drought-selected plants were mostly due to positive complementarity effects (Fig. 2). Of twelve drought-selected species pairs with sufficient replication for separate statistical tests per pair, eleven had positive complementarity effects and five of these were significant $(\mathrm{P}<0.05$; Fig. $2 \mathrm{p})$; whereas ten had negative sampling effects, and six of these were statistically significant $(\mathrm{P}<0.05$; Fig. 2r). In contrast, of eleven ambient-selected species pairs with sufficient replication for separate statistical analyses, six had negative complementarity effects and one of these was significant $(\mathrm{P}<0.05$; Fig. 2o); whereas only two pairs had significantly negative sampling effects $(\mathrm{P}<0.05$; Fig. $2 \mathrm{q})$.

When we used all species pairs in a combined statistical analysis, testing the overall drought selection effect against the variation among species pairs as error term, we found that drought selection had increased post-drought net biodiversity effects in mixtures independent of the particular species pair considered and could thus be generalized across the entire experiment (Fig. 3; Supplementary Table 3). Furthermore, drought-selected species pairs consistently had more positive complementarity and more negative sampling effects than ambient-selected species pairs after the drought event (Fig. 3f, i; Supplementary Table 3). These results suggest that drought-selected plants are more complementary between species than ambient- 
selected plants in mixtures. However, before and during the drought, the effects of drought selection were not statistically significant (Fig. 3; Supplementary Table 3). Initially, the positively biodiversity effect was mainly due to positive sampling effect (Fig. 3a, d, g; Supplementary Table 2).

\section{Effects of drought selection on stability}

Drought-selected plants recovered faster from the drought event in the glasshouse than those of ambient-selected plant; however, this was only evident when plants were grown in mixtures but not in monocultures (Supplementary Fig. 2; Supplementary Table 4). These results suggest that species interactions in mixtures may play important roles in promoting the recovery of drought-selected plants. Plants with different selection treatments did not differ significantly in their resistance or resilience to the drought event neither in mixtures nor in monocultures (Supplementary Fig. 2; Supplementary Table 4).

The difference in recovery rates between mixtures and monocultures (i.e., biodiversity effects on recovery) for drought-selected plants were more positive than those for ambient-selected plants (Fig. 4b; Supplementary Table 5). This is consistent with the more positive biodiversity effects on productivity for drought-selected than ambient-selected plants after the drought event described in the previous section. However, biodiversity effects on resistance were more negative for drought-selected than for ambient-selected plants, thus leading to similar biodiversity effects on resilience between the two selection treatments (Fig. 4a, c).

\section{Effects of drought selection on plant interactions}

The neighbor interaction index $\mathrm{NInt}_{\mathrm{M}}{ }^{49}$, which measures interaction intensity among 
neighboring plants, was mostly negative (Supplementary Fig. 3), indicating that individual plants in pots with four individuals had less biomass than individual plants in pots with one individual. Nevertheless, in most cases average individuals in pots of four individuals had more than $25 \%$ of the biomass of individuals in pots with one individual, that is $\mathrm{NInt}_{\mathrm{M}}>-0.75$, the value expected under the reciprocal yield law ${ }^{50}$. An exception were monocultures after the drought event, which produced the same amount of biomass per pot independent of the number of plants $\left(\mathrm{NInt}_{\mathrm{M}} \approx-0.75\right.$; Supplementary Fig. 3f). Indications of facilitation, i.e., cases where individual plants in pots with four individuals had more biomass than individual plants in pots with one individual and $\mathrm{NInt}_{\mathrm{M}}>0$, were very rare.

During the drought phase in the glasshouse, drought-selected plants competed more strongly in mixtures than ambient-selected plants (Supplementary Fig. 3c;

Supplementary Table 6), which was also the time when drought-selected plants tended to have lower complementarity than ambient-selected plants (Fig. 3e). After the drought event, drought-selected plants showed weaker heterospecific than conspecific competition, which was less pronounced for ambient-selected plants (Figs. 5 \& 6; Supplementary Tables $7 \& 8$ ), consistent with the positive net biodiversity effects on productivity and recovery reported above for drought-selected plants after the drought event. These results suggest that drought-selected plants compared with ambientselected plants had evolved reduced interspecific relative to intraspecific competition (i.e., increased niche differentiation), at least when growing in mixtures and after a novel drought event.

\section{Effects of drought selection on plant traits}

We measured six traits that were closely related to plant water or carbon use on plants 
from pots with one individual. Drought- and ambient-selected plants had similar average values of leaf relative chlorophyll content, leaf area (LA), leaf mass per area (LMA) and leaf osmotic potential before the drought event in the glasshouse (Supplementary Fig. 4). Species varied in their responses of LMA to the selection treatments (Supplementary Table 9). The drought event in the glasshouse reduced leaf stomatal conductance (Supplementary Fig. 5). However, leaf stomatal conductance did not vary significantly between the two selection treatments, neither before nor during the drought event (Supplementary Fig. 5). These results suggest that the drought selection treatments did not lead to significant changes in average values of leaf traits on plants grown alone, at least before the drought event. After the drought phase in the glasshouse, the time window for the biomass harvest after the drought did not allow us to take additional trait measurements. However, drought- and ambientselected plants developed similar root-shoot biomass ratios after the drought (Supplementary Fig. 6).

We also measured leaf relative chlorophyll content, LA and LMA on plants in mixtures before the drought event in the glasshouse. Drought-selected plants diverged in LA between species pairs in mixtures when compared with the same species pairs of ambient-selected plants, although this difference was only marginally significant (Supplementary Fig. 7, Supplementary Table 10). However, species dissimilarities in LMA, leaf relative chlorophyll content or the three traits jointly did not vary significantly between the two selection treatments (Supplementary Fig. 7, Supplementary Table 10).

\section{DISCUSSION}

Increasing threats from extreme climatic events such as droughts have raised the 
importance of predicting ecosystem responses and adaptation to climate change both in short and long terms, based on ecological and evolutionary theory ${ }^{3-6,12-14}$. In this study, we tested whether recurrent summer droughts caused evolutionary adaptations and assessed the effects of drought selection on biodiversity effects for 21 pairs of plant species over the time course of an experimental drought event (before, during and after drought). We found that an 8-year treatment of recurrent droughts had selected for increased niche differentiation in mixtures during the recovery phase after the drought event, which led to more positive biodiversity effects on community productivity and recovery after the drought event. These findings suggest that mixedspecies grassland communities bear the potential of evolutionary adaptations at the community level to extreme climatic events.

We found that drought selection caused a significant difference (relative to ambient selection) in biodiversity effects on productivity only after the drought event in the glasshouse. The selection for more positive biodiversity effects after the drought event was primarily due to the selection for more positive complementarity effects, whereas sampling effects were more negative than those of species pairs selected under ambient conditions. This selection for increased complementarity effects after the drought event was primarily the results of reduced interspecific relative to intraspecific competition (i.e., increased niche differentiation). In contrast to a previous grassland experiment with plants from the Jena Experiment selected under ambient conditions ${ }^{44}$ and in contrast to predictions of the stress-gradienthypothesis (SGH) ${ }^{51}$, we did not find evidence for facilitation underpinning complementarity effects. According to the SGH, interactions among plants are more facilitative in stressful environments where resources are harder to access, but more competitive in environments with a low stress level and a high supply of nutrients ${ }^{51}$. 
However, facilitation, both within and between species, was very rare over the time course of the drought event in our case. During the most stressful phase of the drought event, drought-selected plants competed more strongly between species than did ambient-selected plants. This suggests that evolutionary processes of species interactions can differ between environmental conditions, and observations under ambient climates may not serve to make appropriate predictions for evolutionary responses of communities to extreme climatic events.

The selection for niche differentiation after the drought event could be related to effects of drought on soil nutrients. For example, droughts can increase leaf litter ${ }^{52}$ and reduce the mobility of soil nutrients, the activity of soil microbes and the rate of litter decomposition ${ }^{53-55}$. These constrained resources can be released after droughts 33,34,54, which increases the potential for niche partitioning among species.

We did not test the relative importance of genetic vs. epigenetic variation in explaining our results, but know from a previous study that adaptive changes that had taken place during the course of an 8-year community selection in the Jena Experiment were almost exclusively genetic, with epigenetic variation closely following genetic variation ${ }^{56}$. We also could not determine which traits ${ }^{18}$ drove the observed selection for niche differentiation after the drought event. For the traits that we measured and analyzed, we did not find significant differences between plants selected under recurrent droughts vs. plants selected under ambient conditions. But we should note that this does not necessarily mean that the recurrent droughts did not select for specific traits or trait variation in response to being grown in mixtures, because most of the traits were measured before the drought event in the glasshouse and for single plants, while the primary effects of drought selection were detected after the drought event and in mixtures compared with monocultures. The effects of 
selection on traits may be more evident in mixed-species communities, if the selection primarily induces evolution of species interactions at the level of the community $37,43,45$. This is partially reflected by the findings that competing species composed of drought-selected plants diverged (relative to ambient-selected plants) in their leaf area before the drought event when they were growing together in mixtures. It is also possible that the drought-induced selection may be more apparent on root traits (e.g., root cortical thickness and hydraulic conductance) with close linkages to plant water acquisition but were not measured in this study ${ }^{32}$.

In line with the findings of drought selection for stronger species complementarity during the recovery phase after the drought event, mixtures of drought-selected plants recovered faster than those of ambient-selected plants, which led to a more pronounced positive biodiversity effect on recovery for drought-selected plants. A previous field experiment ${ }^{23}$, where we collected the seeds for the present study, showed that more diverse communities were better able to compensate for drought-driven productivity loss, which led to stabilizing effects of biodiversity. The results from this study suggest that biodiversity-dependent evolutionary adaptation may be an important mechanism driving the above compensatory recovery observed in the field. Biodiversity effects on resistance were more negative for drought-selected than for ambient-selected plants, thus leading to similar biodiversity effects on resilience between the two selection treatments. These findings suggest that droughtselected plants competed more strongly between species than did ambient-selected plants during the drought event, which might have impeded the resistance of mixtures relative to monocultures. Plants of the two selection treatments had similar resistance both in monocultures and mixtures. One explanation for this result could be that the recurrent summer droughts in the field did not cause severe mortality immediately 
during the droughts ${ }^{57}$; thus, the importance of selection for fast recovery from droughts may overwhelm that of selection for high tolerance to droughts. The selection of acquisitive traits for fast recovery after droughts may have side effects on the processes during droughts, for example, intensifying species competition during droughts. Alternatively, the selection processes during droughts may have primarily played out below ground, which we only assessed by measuring biomass allocation to belowground organs in monocultures post drought. We expected it to be increased in drought-selected plants but could not statistically confirm it. A more thorough test of water acquisition ${ }^{32}$.

Predicting the evolution of species interactions and their consequences for ecosystems is challenging due to the prevalent and complex correlations between traits, and trade-offs between survival during extreme events and growth after events $13,33,35,37,58,59$. These complexities imply that a big picture over the time course of an extreme climatic event, integrating both resistance and recovery processes, is necessary for predicting the responses and adaptations of ecosystems to extreme climatic events ${ }^{10,20,23}$.

Drought- and ambient-selected plants had similar biodiversity effects on productivity before the drought event in the glasshouse. Drought-induced selection for niche differentiation after the drought event was not correlated with more positive biodiversity effects before the drought event, although both phases were under ambient conditions with regular watering. This difference may arise from at least the following two reasons. First, drought-induced selection may involve components of plasticity ${ }^{17,60}$. In this case, the effects of selected niche differentiation were only visible when plants were stressed by water deficits, but weak or absent without water 
stress. Second, the resource conditions may have differed between the two phases, which might have affected the available niche axes for partitioning ${ }^{17,61}$. The productivity of mixtures before the drought event (quarter quantiles: [0.148 $\mathrm{g} /$ pot/week, $0.308 \mathrm{~g} /$ pot/week]) were about two times higher than that after the drought event ([0.048 g/pot/week, $0.100 \mathrm{~g} /$ pot/week]). Partitioning along axes associated with light and aboveground space may have been more important before the drought event, while partitioning along axes associated with belowground resources may have been more important after the drought event.

We demonstrated that biodiversity could buffer the impacts of an extreme drought event on ecosystem functioning through selected niche differentiation during the recovery phase post drought. Together with previous findings focusing on ecological processes ${ }^{20-24}$, we add an important mechanism, the evolution of species complementarity, to explain the stability of productivity in mixed-species grassland communities facing climate change. The selection of phenotypes with high recovery after recurrent droughts causes compensatory recovery after drought-related biomass loss. Our results suggest that biodiversity can buffer the impacts of extreme climatic events through evolution of species complementarity and that preserving or restoring biodiversity may increase the potential of evolutionary adaptations to climatic extremes at the community level.

\section{METHODS}

\section{Experimental design}

To test whether an 8-year selection treatment of recurrent summer droughts would change biodiversity effects and species interactions of grassland plants when facing a novel drought event, we grew ambient- vs. drought-selected plants of 12 species in a 
glasshouse. The plants were grown from seeds collected from mixed-species communities under 8-year treatments of yearly summer droughts vs. ambient precipitation in a biodiversity field experiment in Jena, Germany ${ }^{23,47}$.

The Jena Experiment was established in 2002 using a common seed pool of 60 grassland species, with $8020 \times 20 \mathrm{~m}$ large plots of species richness levels of 1, 2, 4, 8, 16 and 60 species ${ }^{46}$. Most of the species are perennial and capable of outcrossing. The Jena Drought Experiment ${ }^{23,47}$ was initiated in 2008 . Two $1 \times 1 \mathrm{~m}$ subplots were set within each large plot, designated as either drought treatment or ambient control. For the drought treatment, rainout shelters were set up to exclude natural rainfall in mid-summer for six weeks. The ambient control treatment got the same shelter construction but rain water was reapplied to not confound the results with artifacts from the shelter ${ }^{62}$. We collected seeds from drought and control subplots throughout the 2016 growing season (Fig. 1). Seeds were collected from mixtures in order to test in the glasshouse the effects of drought-induced selection on species interactions, and because seeds of only two species were available from monocultures. Although we obtained seeds of 17 species, we only used 12 of them, because the other five species had either few seeds or low germination rates. The 12 plant species represented four functional groups (grass, small herb, tall herb, and legume) (Supplementary Table 1) 46.

We planted the seedlings in February 2017 in a glasshouse (day temperature range $20-25^{\circ} \mathrm{C}$, night temperature range $15-21^{\circ} \mathrm{C}$, and humidity range $60-80 \%$ ) at the University of Zurich, Switzerland. Seeds from drought and ambient subplots of the same large plot in Jena were sown individually, in monocultures or in 2-species mixtures in the glasshouse (Fig. 1). Plants of the three community treatments (individual, monoculture and mixture) were from the same seed families as far as 
possible. For those without perfect matches in seed families, plants at least originated from Jena subplots with the same plant functional group composition and selection treatment. In the glasshouse experiment, both monocultures and mixtures contained four plants within a pot. The pots were $11 \times 11 \times 11.5 \mathrm{~cm}$ in size and filled with soil composed of $50 \%$ collected from a sugar-beet field, $25 \%$ sand and $25 \%$ perlite. We randomly assigned the pots into four blocks in the glasshouse. To test the effects of drought-induced selection on plant traits, we sowed individual plants of the 12 species in a fifth block. Within the first two weeks, dead individuals were replaced, thereafter dead individuals were not replaced anymore. In total, we established 958 pots: 257 pots of mixtures, 217 pots of monocultures and 484 pots of individual plants ( 244 pots of individuals in blocks $1-4$, and 240 pots of individuals in block 5). For mixtures, there were 21 species pairs (Supplementary Table 1). Species pairs including Crepis biennis or Lotus corniculatus as one partner had low numbers of replicates (Supplementary Table 1). However, including or excluding these communities produced qualitatively similar results. Thus, we present the results including these two species in this paper (Figs. 3, 4 and 6).

During a first phase of three month in the glasshouse (Fig. 1), pots were watered regularly ("before drought"). After 14-18 weeks, when most of the species had reached peak aboveground biomass, we harvested them (first harvest). This was followed by a second phase of two weeks without watering ("during drought"). Soil moisture decreased from more than $40 \%$ to less than $10 \%$ after 10 days since the drought initiation. During a third phase of seven weeks, pots were watered regularly again for recovery until most plants reached a new aboveground biomass peak again (“after drought”). We conducted three harvests: 14-18 weeks (before the drought event), 20 weeks (at the end of the drought event) and 27 weeks (after complete 
recovery from the drought event) after establishment of the experiment in the glasshouse. At each harvest, we cut the plants at about $3 \mathrm{~cm}$ above soil and dried the harvested plant material at $70^{\circ} \mathrm{C}$ for 48 hours to obtain the dry biomass. We refer to the three harvests as before, during and after drought, and use the aboveground biomass as a proxy for productivity.

\section{Additive partitioning}

We used the additive partitioning approach ${ }^{48}$ to decompose the net biodiversity effect (NE) on aboveground biomass into complementarity effect (CE) and sampling effect (SE), separately for each harvest, selection treatment and block. We did not perform the partitioning for mixtures with zero biomass ${ }^{63}$. For monocultures with zero biomass, we kept the ones which had alive plants in the previous harvest but excluded the ones which had already lost all of them. For example, when we did the partitioning for the second harvest, we kept the monocultures that had zero biomass in the second harvest but non-zero biomass in the first harvest; we excluded the monocultures that had zero biomass already in the first harvest. This was to assure that communities that died before the drought could not reappear during or after the drought, and communities that had died during the drought could not reappear after the drought.

We used mixed-effects models to assess the influences of drought vs. ambient selection treatments on biodiversity effects separately for each harvest (Fig. 3, Supplementary Table 3). Block and selection treatment were set as fixed-effects terms, while species composition (identity of species pair) and its interaction with selection treatment were set as random-effects terms. This conservative approach was used to allow for generalizations across all possible species compositions, although a more 
liberal approach with species composition and its interactions as fixed-effects terms could also been applied (see Schmid et al. $2017^{64}$ for a discussion of defining terms as fixed- vs. random-effects terms, including a justification of preference for treating block as fixed-effects term). We square-root transformed the CEs and SEs with sign reconstruction $(\operatorname{sign}(y) \sqrt{y})$ prior to analysis to improve the normality of residuals ${ }^{48}$. The mixed-effects model did not converge in the analysis with CE after the drought event. In this case, we used a general linear model, in which we fitted block, species composition, selection treatment, and species composition by selection treatment interaction in this order. Then we tested the significance of selection treatment using its interaction with species composition as error term. This procedure is an alternative to mixed-effects models that estimate variance components for random-effects terms with maximum likelihood ${ }^{64}$.

We additionally tested the significance of biodiversity effects on productivity separately for each selection treatment and harvest (Supplementary Table 2). We set block and species composition as fixed- and random-effects terms, respectively. The model corresponding to $\mathrm{CE}$ for ambient-selected plants during the drought event did not converge so that we fitted it with a general linear model, in which we tested the significance of overall mean (intercept) using species composition as error term. All statistical analyses were conducted in R $3.6 .3^{65}$. The mixed-effects models were conducted with asreml-R package 4.1.0.110 ${ }^{66}$.

Finally, we also tested whether effects of drought selection on biodiversity effects in the glasshouse depended on the functional group richness (number of functional groups: 1, 2, 3 or 4) in the Jena field plots, but could not detect any significant trend (Supplementary Table 11). Therefore, we excluded the history of functional group richness from further analyses and presentation in this paper. 
To assess the temporal responses of community aboveground biomass to the drought event, we calculated three indices representing different facets of stability: biomass resistance, recovery and resilience (see van Moorsel et al. $2021{ }^{19}$ for an example). We calculated resistance as the biomass ratio during vs. before the drought, recovery as the ratio after vs. during the drought and resilience as the ratio after vs. before the drought (see also Isbell et al. $2015^{20}$ ). We log-transformed the indices (plus a half of the minimum positive value to allow taking logs of indices that were originally zero) prior to statistical analyses to improve the normality of residuals. Excluding index values that were originally zero produced qualitatively similar results. To assess the effects of drought selection on biomass stability, we fitted mixedeffects models with block and selection treatment as fixed-effects terms, and species composition and its interaction with selection treatment as random-effects terms (Supplementary Fig. 2, Supplementary Table 4). We fitted the models separately for mixtures and monocultures. We included the log-transformed biomass at the first harvest as a covariate because biomass stability in response to droughts often depends on plant performance under ambient conditions.

In the same way as net biodiversity effects on productivity were calculated for additive partitioning, we calculated biodiversity effects on biomass stability as the difference between each mixture and its corresponding monocultures. Then, we tested the influence of selection treatment on the biodiversity effects on biomass stability. Block and selection treatment were set as fixed-effects terms; species composition and its interaction with selection treatment were set as random-effects terms (Fig. 4, Supplementary Table 5). The log-transformed biomass at the first harvest was also 
included as a covariate ${ }^{19}$. To assess the significance of biodiversity effects on biomass stability for each selection treatment, we fitted another set of simplified models, with block and log-transformed biomass as fixed-effects terms, and species composition as random-effects term (Fig. 4).

\section{Neighbor interactions}

We assessed interactions between neighboring plants within pots using the metrics of neighbor interaction intensity with multiplicative symmetry $\left(\mathrm{NInt}_{\mathrm{M}}\right)^{49}$ :

$$
\operatorname{NInt}_{M}=2 \frac{\Delta P}{P_{-N}+P_{+N}+|\Delta P|}
$$

where $P_{-N}$ and $P_{+N}$ are the productivities without (individual plant) and with neighbors (monocultures or mixtures), respectively; $\Delta P=P_{+N}-P_{-N}$. Negative values of $\mathrm{NInt}_{M}$ indicate competition and positive values indicate facilitation. $\mathrm{NInt}_{M}$ is bounded between -1 (competitive exclusion) and 1 ("obligate" facilitation). For monocultures, we first calculated the per-plant biomass as the ratio between total biomass and planting density, and then used the per-plant value to compare with the corresponding individuals (without neighbor) of the same species with the same selection treatment in the same block. Note that under the reciprocal yield law ${ }^{50}$, an individual grown alone in a pot should be four times larger than an individual grown with three others in a pot, resulting in a $\mathrm{NInt}_{\mathrm{M}}$ of -0.75 . For 2 -species mixtures, we calculated the per-plant biomass separately for each species and took the average $\mathrm{NInt}_{\mathrm{M}}$ of the two species to measure the interaction intensity of the mixture. We set zero biomass for dead plants in the calculation. Again, if mixtures would also follow the reciprocal yield law independent of species identity, then $\mathrm{NInt}_{\mathrm{M}}=-0.75$ would be expected. Values greater than -0.75 indicate some sort of overyielding due to higher density or higher density and higher diversity. 

tested the effects of selection treatments on neighbor interaction intensity separately for monocultures and mixtures. We included block and selection treatment as fixedeffects terms, species composition and its interaction with selection treatment as random-effects terms (Supplementary Fig. 3, Supplementary Table 6). conspecific interactions in its two corresponding monocultures. A positive value of this difference indicates a weaker heterospecific than conspecific competition (i.e., niche differentiation) or stronger heterospecific than conspecific facilitation, which may lead to a positive complementarity effect. We tested the effects of selection treatments on interaction difference for each harvest by fitting block and selection treatment as fixed-effects terms, and species composition and its interaction with selection treatment as random-effects terms (Fig. 6, Supplementary Table 8). We also tested the significance of the interaction difference for each selection treatment by fitting block and species composition as fixed- and random-effects term, respectively

525 (Fig. 6, Supplementary Table 7).

\section{Plant traits}

To assess whether drought selection would change plant traits, we measured six traits (Supplementary Table 9), which are closely related to plant usages of water or carbon, on plants in pots with one individual from blocks $1-5$. We focused on the traits on individual plants without neighbor to evaluate the influence of selection treatment on traits without the impacts of plasticity induced by plant interactions. We measured leaf relative chlorophyll content, leaf area (LA), leaf mass per area (LMA) and leaf osmometric pressure before the drought; leaf stomatal conductance both before and 
during the drought; and dry biomass ratio between root and shoot after the drought (in the third harvest). Leaf relative chlorophyll content was measured for three mature, fully expanded leaves per plant by using a SPAD-502 Plus chlorophyll meter from Konica Minolta. LA was obtained by scanning 3-4 mature, fully expanded leaves per plant with a LI-3100C Area Meter from LI-COR. LMA was calculated as the ratio between leaf dry mass (oven dried at $70^{\circ} \mathrm{C}$ for 48 hours, using the same leaves that for LA) and LA. Leaf osmotic potential at full hydration was considered as an important trait associated with plant tolerance to drought ${ }^{67}$. We measured leaf osmotic potential with freeze-thaw leaf pieces cut from 1-2 mature, fully expanded leaves per plant by using a Wescor vapor pressure osmometer VAPRO (Model 5520) according to the method by Bartlett, et al. ${ }^{67}$. Plants were fully hydrated one day before the leaf sampling for osmotic potential measurement. Leaf stomatal conductance is a measure of exchange rate of carbon dioxide and water vapor through the stomata ${ }^{68}$. It was measured for 3-5 healthy mature leaves per plant by using a SC-1 Leaf Porometer from Decagon Devices. For grass species, 3 blades were placed adjacent to each other in order to have a large enough area for the measurement of stomatal conductance.

For stomatal conductance during the drought event, we measured the individual plants from block 5 only due to limited time during the drought phase. We harvested aboveground and belowground plant biomass separately for alive individual plants at the end of the experiment (after the complete recovery from the drought). The ovendried $\left(70^{\circ} \mathrm{C}\right.$ for 48 hours) aboveground and belowground biomass was used to calculate the biomass ratio between root and shoot. We took the average value of each trait of each plant for statistical analyses.

We used linear mixed-effects models to assess the influence (generalized across species) of selection treatments on trait values (red lines in Supplementary Figs. 4-6). 
Block and selection treatment were set as fixed-effects terms; species and its interaction with selection treatment were set as random-effects terms. Alternatively, we set species, selection treatment and their interaction as fixed-effects terms to assess whether species responded differently to the selection treatments (Supplementary Table 9). LMA, LA, leaf osmotic potential, leaf stomatal conductance and root-shoot biomass ratio were log-transformed to improve normality of residuals.

$$
\text { We also measured leaf relative chlorophyll content, LA and LMA in mixtures }
$$
before the drought event (Supplementary Table 10) to evaluate the influence of selection treatments on trait dissimilarity between interacting species within communities. We calculated the absolute trait distance between two species in each mixture both separately for each trait and jointly with the three traits. For multi-traitbased dissimilarity, we calculated the Euclidean trait distance in standardized threedimensional trait space.

We used linear mixed-effects models to assess the effects of selection treatments on trait dissimilarity in mixtures (Supplementary Table 10). Block and selection treatment were set as fixed-effects terms; species composition and its interaction with selection treatment were set as random-effects terms. The model for LA dissimilarity did not converge so that we fit it with a general linear model, in which we tested the significance of selection treatment using its interaction with species composition as error term. For the models with LA, LMA and the joint three traits as dependent variables, we removed one pot (B1P674) because the LA value of Alopecurus pratensis in this pot was extremely small (about $1 / 3$ of the second minimum value of the same species in mixtures). However, including or excluding this pot produced qualitatively similar results. 
Author Contribution: M.S.-L and A.W. conceived the Jena Drought Experiment, Y.C., B.S., A.V. and C.W. conceived the glasshouse experiment to test selection effects. Y.C., M.I.G., C.W. and T.X. carried out the glasshouse experiment. Y.C., B.S. and A.V. developed the analytical procedure, and Y.C. did the statistical analyses. All authors discussed the results and helped write the paper.

Data Availability: The data supporting the findings of this study are available at the Figshare digital repository: https://figshare.com/s/27107d80d374be6fe6c4.

Code Availability: The code supporting the results are available at the Figshare digital repository: https://figshare.com/s/8fdfb27d224e47f1a8e6.

Competing Interest: The authors declare no competing interest.

\section{Acknowledgements:}

Y.C. acknowledges support from National Natural Science Foundation of China (32071536 and 31700349). B.S. was supported by the Swiss National Science Foundation (grant number 31003A_166457) and the University of Zurich Research Priority Program on Global Change and Biodiversity. N.E. acknowledges support from the German Centre for Integrative Biodiversity Research (iDiv) Halle-JenaLeipzig, funded by the German Research Foundation (FZT 118). The Jena Experiment is funded by the Deutsche Forschungsgemeinschaft (DFG, German Research Foundation, FOR 456, FOR 1451, and FOR 5000). We thank Gerlinde Kratsch, Ulrike Gudd and the gardeners of the Jena Experiment for help with the selection experiment.

\section{REFERENCES}

1 Dai, A. Increasing drought under global warming in observations and models. 
Nature Climate Change 3, 52-58, doi:10.1038/nclimate1633 (2012).

2 IPCC. Summary for Policymakers. Global Warming of $1.5^{\circ} \mathrm{C}$. An IPCC Special Report on the impacts of global warming of $1.5^{\circ} \mathrm{C}$ above preindustrial levels and related global greenhouse gas emission pathways, in the context of strengthening the global response to the threat of climate change, sustainable development, and efforts to eradicate poverty (World Meteorological Organization, 2018).

3 Vicente-Serrano, S. M. et al. Response of vegetation to drought time-scales across global land biomes. Proceedings of the National Academy of Sciences 110, 52-57, doi:10.1073/pnas.1207068110 (2012).

4 Brodribb, T. J., Powers, J., Cochard, H. \& Choat, B. Hanging by a thread? Forests and drought. Science 368, 261-266, doi:10.1126/science.aat7631 (2020).

5 Anderegg, W. R. L. et al. Climate-driven risks to the climate mitigation potential of forests. Science 368, eaaz7005, doi:10.1126/science.aaz7005 (2020).

6 Reichstein, M. et al. Climate extremes and the carbon cycle. Nature 500, 287295, doi:10.1038/nature12350 (2013).

7 Anderegg, W. R. L., Kane, J. M. \& Anderegg, L. D. L. Consequences of widespread tree mortality triggered by drought and temperature stress. Nature Climate Change 3, 30-36, doi:10.1038/nclimate1635 (2012).

8 Engelbrecht, B. M. J. et al. Drought sensitivity shapes species distribution patterns in tropical forests. Nature 447, 80-82, doi:10.1038/nature05747 (2007).

9 Fauset, S. et al. Drought-induced shifts in the floristic and functional 
composition of tropical forests in Ghana. Ecology Letters 15, 1120-1129, doi:10.1111/j.1461-0248.2012.01834.x (2012).

10 McDowell, N. G. et al. Pervasive shifts in forest dynamics in a changing world. Science 368, eaaz9463, doi:10.1126/science.aaz9463 (2020).

11 Franks, S. J., Sim, S. \& Weis, A. E. Rapid evolution of flowering time by an annual plant in response to a climate fluctuation. Proceedings of the National Academy of Sciences 104, 1278-1282, doi:10.1073/pnas.0608379104 (2007).

12 Grant, P. R. et al. Evolution caused by extreme events. Philosophical Transactions of the Royal Society B: Biological Sciences 372, 20160146, doi:10.1098/rstb.2016.0146 (2017).

13 Gutschick, V. P. \& BassiriRad, H. Extreme events as shaping physiology, ecology, and evolution of plants: toward a unified definition and evaluation of their consequences. New Phytologist 160, 21-42, doi:10.1046/j.14698137.2003.00866.x (2003).

14 Hoffmann, A. A. \& Sgrò, C. M. Climate change and evolutionary adaptation. Nature 470, 479-485, doi:10.1038/nature09670 (2011).

15 Ravenscroft, C. H., Whitlock, R. \& Fridley, J. D. Rapid genetic divergence in response to 15 years of simulated climate change. Global Change Biology 21, 4165-4176, doi:10.1111/gcb.12966 (2015).

16 Shaw, R. G. \& Etterson, J. R. Rapid climate change and the rate of adaptation: insight from experimental quantitative genetics. New Phytologist 195, 752765, doi:10.1111/j.1469-8137.2012.04230.x (2012).

17 Avolio, M. L. \& Smith, M. D. Mechanisms of selection: Phenotypic differences among genotypes explain patterns of selection in a dominant species. Ecology 94, 953-965, doi:10.1890/12-1119.1 (2013). 
65918 Metz, J. et al. Rapid adaptive evolution to drought in a subset of plant traits in

a large-scale climate change experiment. Ecolgy Letters 23, 1643-1653, doi:10.1111/ele.13596 (2020).

664 and resilience in experimental plant communities. Ecology 102, e03205,

20 Isbell, F. et al. Biodiversity increases the resistance of ecosystem productivity to climate extremes. Nature 526, 574-577, doi:10.1038/nature15374 (2015).

Anderegg, W. R. L. et al. Hydraulic diversity of forests regulates ecosystem resilience during drought. Nature 561, 538-541, doi:10.1038/s41586-0180539-7 (2018).

22 O’Brien, M. J., Reynolds, G., Ong, R. \& Hector, A. Resistance of tropical

23 Wagg, C. et al. Plant diversity maintains long-term ecosystem productivity under frequent drought by increasing short-term variation. Ecology 98, 29522961, doi:10.1002/ecy.2003 (2017). 367, 363-365, doi:10.1038/367363a0 (1994).

67825 Tilman, D., Lehman, Clarence L. \& Bristow, Charles E. Diversity-Stability Relationships: Statistical Inevitability or Ecological Consequence? The American Naturalist 151, 277-282, doi:10.1086/286118 (1998). stability: a synthesis of underlying mechanisms. Ecology Letters 16 Suppl 1, 106-115, doi:10.1111/ele.12073 (2013). 
27 Barrett, R. \& Schluter, D. Adaptation from standing genetic variation. Trends in Ecology \& Evolution 23, 38-44, doi:10.1016/j.tree.2007.09.008 (2008).

28 Fakheran, S. et al. Adaptation and extinction in experimentally fragmented landscapes. Proceedings of the National Academy of Sciences 107, 1912019125, doi:10.1073/pnas.1010846107 (2010).

29 Sun, S., Jung, E., Gaviria, J. \& Engelbrecht, B. M. J. Drought survival is positively associated with high turgor loss points in temperate perennial grassland species. Functional Ecology 34, 788-798, doi:10.1111/1365$2435.13522(2020)$.

30 Greenwood, S. et al. Tree mortality across biomes is promoted by drought intensity, lower wood density and higher specific leaf area. Ecology Letters 20, 539-553, doi:10.1111/ele.12748 (2017).

31 Olson, M. E. et al. Plant height and hydraulic vulnerability to drought and cold. Proceedings of the National Academy of Sciences 115, 7551-7556, doi:10.1073/pnas.1721728115 (2018).

32 Freschet, G. T. et al. Root traits as drivers of plant and ecosystem functioning: current understanding, pitfalls and future research needs. New Phytologist, doi:10.1111/nph.17072 (2021).

33 Mackie, K. A., Zeiter, M., Bloor, J. M. G., Stampfli, A. \& de Vries, F. Plant functional groups mediate drought resistance and recovery in a multisite grassland experiment. Journal of Ecology 107, 937-949, doi:10.1111/13652745.13102 (2019).

34 Gessler, A., Schaub, M. \& McDowell, N. G. The role of nutrients in droughtinduced tree mortality and recovery. New Phytologist 214, 513-520, doi:10.1111/nph.14340 (2016). 
35 Goodnight, C. J. Experimental Studies of Community Evolution II: The Ecological Basis of the Response to Community Selection. Evolution 44, 1625-1636, doi:10.2307/2409342 (1990).

36 terHorst, C. P. et al. Evolution in a Community Context: Trait Responses to Multiple Species Interactions. The American Naturalist 191, 368-380, doi:10.1086/695835 (2018).

37 Whitham, T. G., Allan, G. J., Cooper, H. F. \& Shuster, S. M. Intraspecific Genetic Variation and Species Interactions Contribute to Community Evolution. Annual Review of Ecology, Evolution, and Systematics 51, 587-612, doi:10.1146/annurev-ecolsys-011720-123655 (2020).

38 Matías, L., Godoy, O., Gómez-Aparicio, L., Pérez-Ramos, I. M. \& Allan, E. An experimental extreme drought reduces the likelihood of species to coexist despite increasing intransitivity in competitive networks. Journal of Ecology 106, 826-837, doi:10.1111/1365-2745.12962 (2018).

39 Godoy, O., Gómez-Aparicio, L., Matías, L., Pérez-Ramos, I. M. \& Allan, E. An excess of niche differences maximizes ecosystem functioning. Nature Communications 11, 4180, doi:10.1038/s41467-020-17960-5 (2020).

40 Turnbull, L. A., Levine, J. M., Loreau, M. \& Hector, A. Coexistence, niches and biodiversity effects on ecosystem functioning. Ecology Letters 16 Suppl 1, 116-127, doi:10.1111/ele.12056 (2013).

41 Fiegna, F., Moreno-Letelier, A., Bell, T. \& Barraclough, T. G. Evolution of species interactions determines microbial community productivity in new environments. The ISME Journal 9, 1235-1245, doi:10.1038/ismej.2014.215 (2014).

42 Lawrence, D. et al. Species Interactions Alter Evolutionary Responses to a 
Novel Environment. PLoS Biology 10, e1001330, doi:10.1371/journal.pbio.1001330 (2012).

43 Zuppinger-Dingley, D. et al. Selection for niche differentiation in plant communities increases biodiversity effects. Nature 515, 108-111, doi:10.1038/nature13869 (2014). requires diverse communities. Nature Ecology \& Evolution 2, 1381-1385, doi:10.1038/s41559-018-0623-2 (2018).

van Moorsel, S. J. et al. Community evolution increases plant productivity at low diversity. Ecology Letters 21, 128-137, doi:10.1111/ele.12879 (2018). Roscher, C. et al. The role of biodiversity for element cycling and trophic
interactions: an experimental approach in a grassland community. Basic and
Applied Ecology 5, 107-121, doi:10.1078/1439-1791-00216 (2004). Roscher, C. et al. The role of biodiversity for element cycling and troph
interactions: an experimental approach in a grassland community. Ba
Applied Ecology 5, 107-121, doi:10.1078/1439-1791-00216 (2004). Vogel, A., Scherer-Lorenzen, M. \& Weigelt, A. Grassland Resistance and Resilience after Drought Depends on Management Intensity and Species Richness. PLoS ONE 7, e36992, doi:10.1371/journal.pone.0036992 (2012). Ecology and Evolution 8, 580-591, doi:10.1111/2041-210x.12706 (2017). biodiversity experiments. Nature 412, 72-76, doi:10.1038/35083573 (2001). Díaz-Sierra, R. et al. A new family of standardized and symmetric indices for measuring the intensity and importance of plant neighbour effects. Methods in Ecology and Evolution 8, 580-591, doi.10.1111/2041-210x.12706 (2017).

50 Harper, J. L. Population Biology of Plants. (Academic Press, 1977).

51 Bertness, M. D. \& Callaway, R. Positive interactions in communities. Trends in Ecology \& Evolution 9, 191-193, doi:10.1016/0169-5347(94)90088-4 (1994). 
52 Wright, S. J. \& Cornejo, F. H. Seasonal drought and leaf fall in a tropical forest. Ecology 71, 1165-1175, doi:10.2307/1937384 (1990).

53 Vogel, A., Eisenhauer, N., Weigelt, A. \& Scherer-Lorenzen, M. Plant diversity does not buffer drought effects on early-stage litter mass loss rates and microbial properties. Global Change Biology 19, 2795-2803, doi:10.1111/gcb.12225 (2013).

54 Hofer, D., Suter, M., Buchmann, N. \& Lüscher, A. Nitrogen status of functionally different forage species explains resistance to severe drought and post-drought overcompensation. Agriculture, Ecosystems \& Environment 236, 312-322, doi:10.1016/j.agee.2016.11.022 (2017).

55 Manzoni, S., Schimel, J. P. \& Porporato, A. Responses of soil microbial communities to water stress: results from a meta-analysis. Ecology 93, 930938, doi:10.1890/11-0026.1 (2012).

56 van Moorsel, S. J. et al. Evidence for rapid evolution in a grassland biodiversity experiment. Molecular Ecology 28, 4097-4117, doi:10.1111/mec.15191 (2019).

57 Korell, L., Auge, H., Chase, J. M., Harpole, S. \& Knight, T. M. We need more realistic climate change experiments for understanding ecosystems of the future. Global Change Biology 26, 325-327, doi:10.1111/gcb.14797 (2019).

58 Wright, I. J. et al. The worldwide leaf economics spectrum. Nature 428, 821827, doi:10.1038/nature02403 (2004).

59 Niinemets, Ü. Is there a species spectrum within the world-wide leaf economics spectrum? Major variations in leaf functional traits in the Mediterranean sclerophyllQuercus ilex. New Phytologist 205, 79-96, doi:10.1111/nph.13001 (2015). 
60 Franks, S. J., Weber, J. J. \& Aitken, S. N. Evolutionary and plastic responses to climate change in terrestrial plant populations. Evolutionary Applications 7, 123-139, doi:10.1111/eva.12112 (2014).

61 Kooyers, N. J., James, B. \& Blackman, B. K. Competition drives trait evolution and character displacement betweenMimulusspecies along an environmental gradient. Evolution 71, 1205-1221, doi:10.1111/evo.13200 (2017).

62 Vogel, A. et al. Separating Drought Effects from Roof Artifacts on Ecosystem Processes in a Grassland Drought Experiment. PLoS ONE 8, e70997, doi:10.1371/journal.pone.0070997 (2013).

63 Clark, A. T. et al. How to estimate complementarity and selection effects from an incomplete sample of species. Methods in Ecology and Evolution 10, 21412152, doi:10.1111/2041-210x.13285 (2019).

64 Schmid, B., Baruffol, M., Wang, Z. \& Niklaus, P. A. A guide to analyzing biodiversity experiments. Journal of Plant Ecology 10, 91-110, doi:10.1093/jpe/rtw107 (2017).

65 R Core Team. R: A Language and Environment for Statistical Computing. Version 3.6.3 (R Foundation for Statistical Computing, Vienna, Austria, 2020).

66 Butler, D. asreml: fits the linear mixed model. R package version 4.1.0.110 (2019).

67 Bartlett, M. K. et al. Rapid determination of comparative drought tolerance traits: using an osmometer to predict turgor loss point. Methods in Ecology and Evolution 3, 880-888, doi:10.1111/j.2041-210X.2012.00230.x (2012).

68 Damour, G., Simonneau, T., Cochard, H. \& Urban, L. An overview of models of stomatal conductance at the leaf level. Plant Cell \& Environment 33, 1419- 

1438, doi:10.1111/j.1365-3040.2010.02181.x (2010).

810 


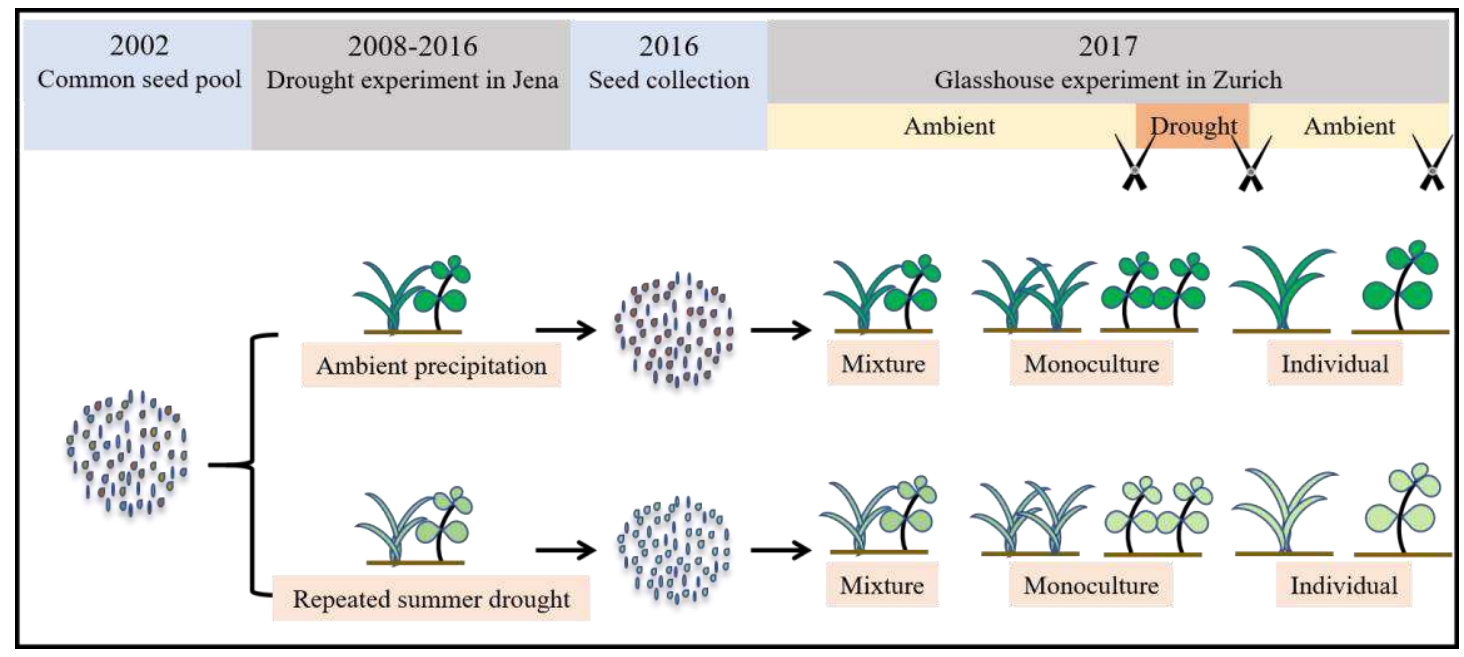

Figure 1 | Experimental design. Seeds collected from different selection treatments

814 (8-years treatments of recurrent summer droughts vs. ambient control) in the Jena

815 Experiment, Germany, were sown in 2-species mixtures, in monocultures or

816 individually in pots in a glasshouse at the University of Zurich, Switzerland. During a

817 first phase of three months, pots were watered regularly (ambient conditions, "before

818 drought"). This was followed by a second phase of two weeks without watering

819 (drought conditions, "during drought") and a third phase of seven weeks with regular

820 watering (ambient conditions for recovery, "after drought"). Plants were harvested

821 after 14-18 weeks (before the drought), 20 weeks (after the drought) and 27 weeks

822 (after recovery from the drought) as represented by scissors. 

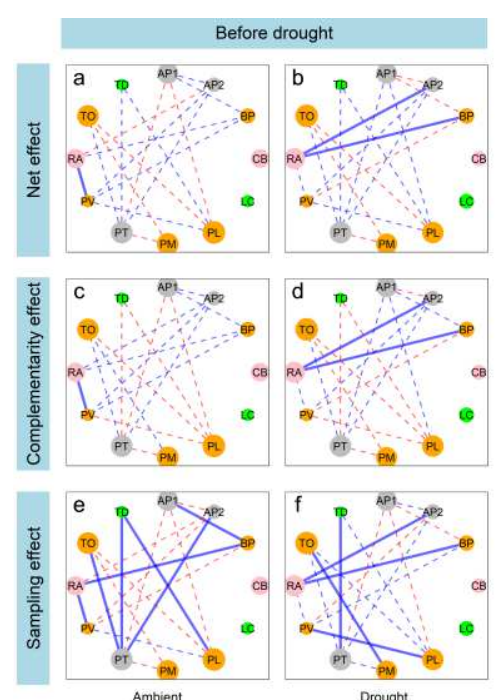

823
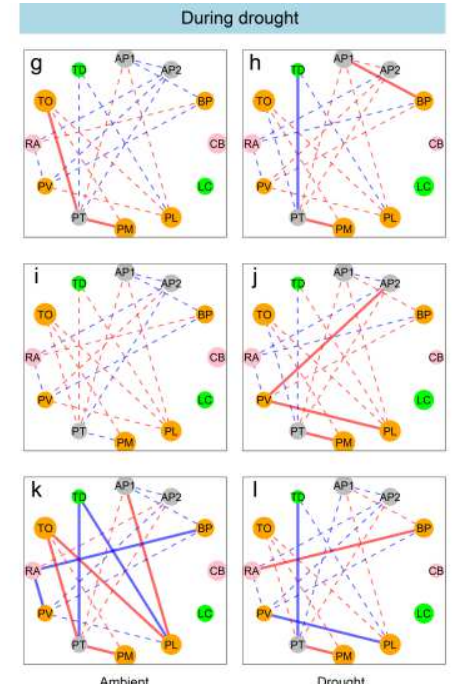

Selection treatment
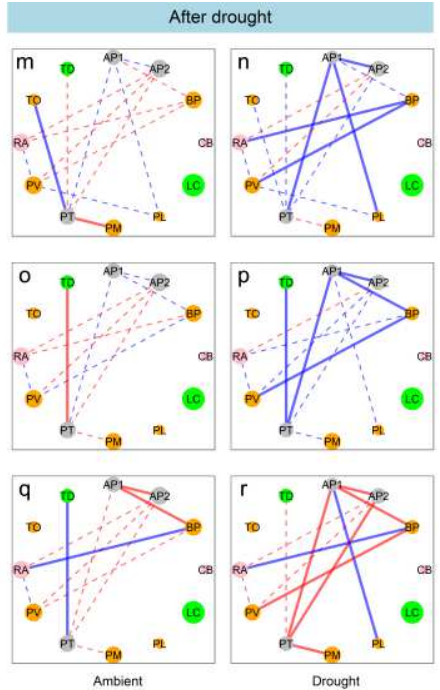

Figure 2 | Effects of biodiversity on productivity among different 2-species mixtures for each selection treatment (ambient- vs. drought-selected plants) and harvest (before $[\mathrm{a}-\mathrm{f}]$, during $[\mathrm{g}-\mathrm{l}]$ and after $[\mathrm{m}-\mathrm{r}]$ the drought event in the glasshouse). Biodiversity effects were calculated as net effect (first row), complementarity effect (second row) and sampling effect (third row). Lines connecting nodes (species) indicate species pairs within mixtures in the glasshouse. Blue and red lines indicate positive and negative average biodiversity effects, respectively, with the solid lines indicating significant effects based on Student's t test $(\alpha=0.05$; mixtures of each selection treatment with less than three replicates at a harvest were not tested for significance and hence not shown in this figure). Node sizes are proportional to the corresponding monoculture biomass in each panel. Node colors indicate different functional groups (green=legume, grey=grass, orange $=$ short herb, pink=tall herb). Full species names are shown in Supplementary Table 1. 

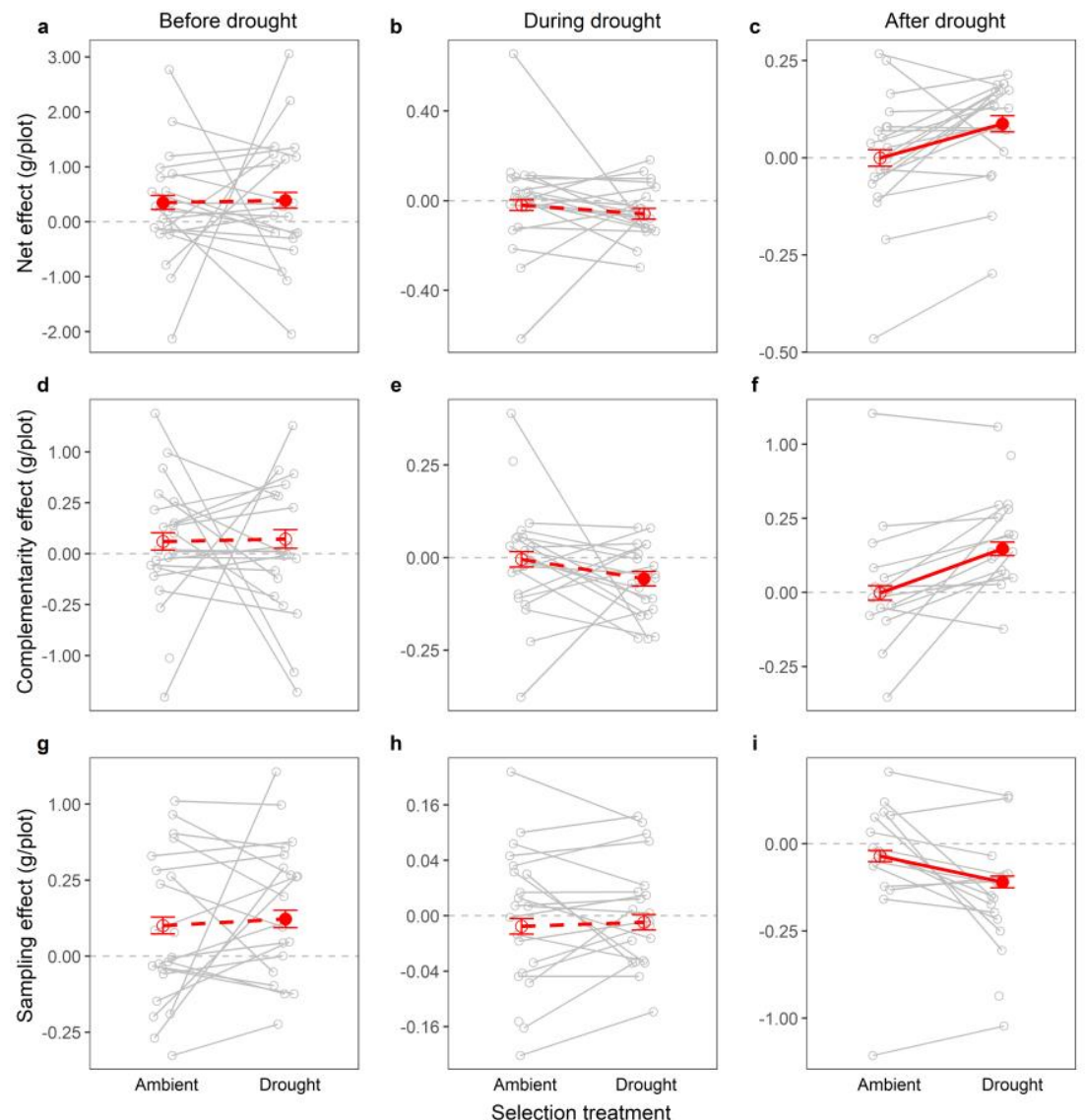

Figure 3 | Difference in biodiversity effects on productivity between selection

treatments (ambient- vs. drought-selected plants) across time (before [first

the glasshouse). Biodiversity effects were calculated as net effect $(\mathrm{a}-\mathrm{c})$, significant differences between the two selection treatments (see Supplementary Table significant differences from zero (horizontal dashed lines, see Supplementary Table connect the same species pair between the two selection treatments. 
851

852

853

854

855

856

857

858

859

860

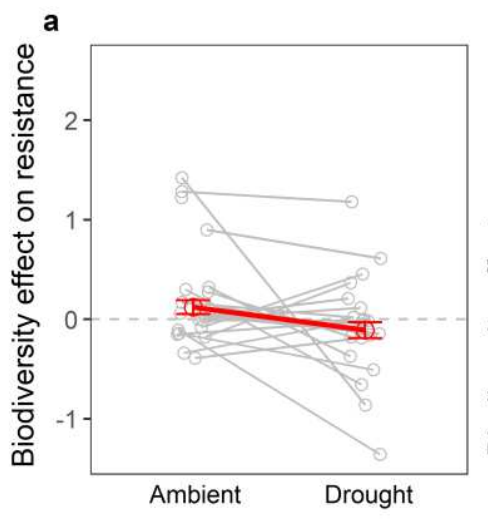

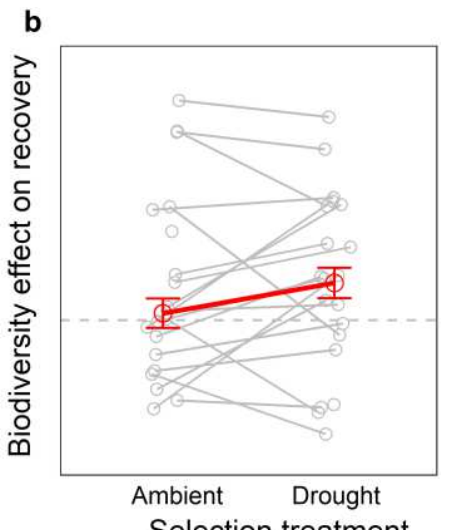

Selection treatment

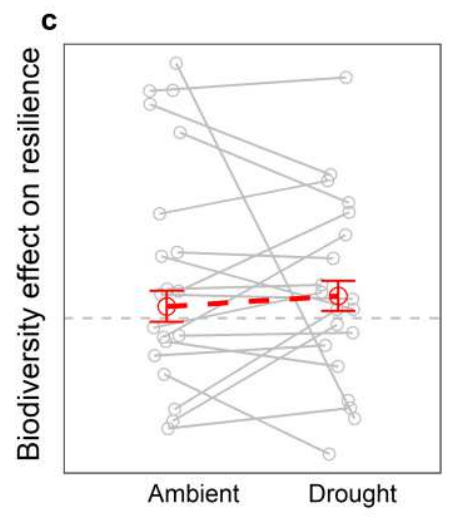

Figure 4 | Difference in biodiversity effects on biomass stability in response to the

drought-selected plants). The solid red lines indicate significant differences between

the two selection treatments (see Supplementary Table 5). Biomass stability was

calculated as resistance (a), recovery (b) and resilience (c). Biodiversity effects were

calculated as the differences in stability indices between mixtures and monocultures.

Red points and error bars show the means \pm standard error of 2-species mixtures

composed of the corresponding selection treatment. Grey points represent means for

species pairs (standard errors for species pairs were not shown). Grey lines connect

the same species pair between the two selection treatments. 

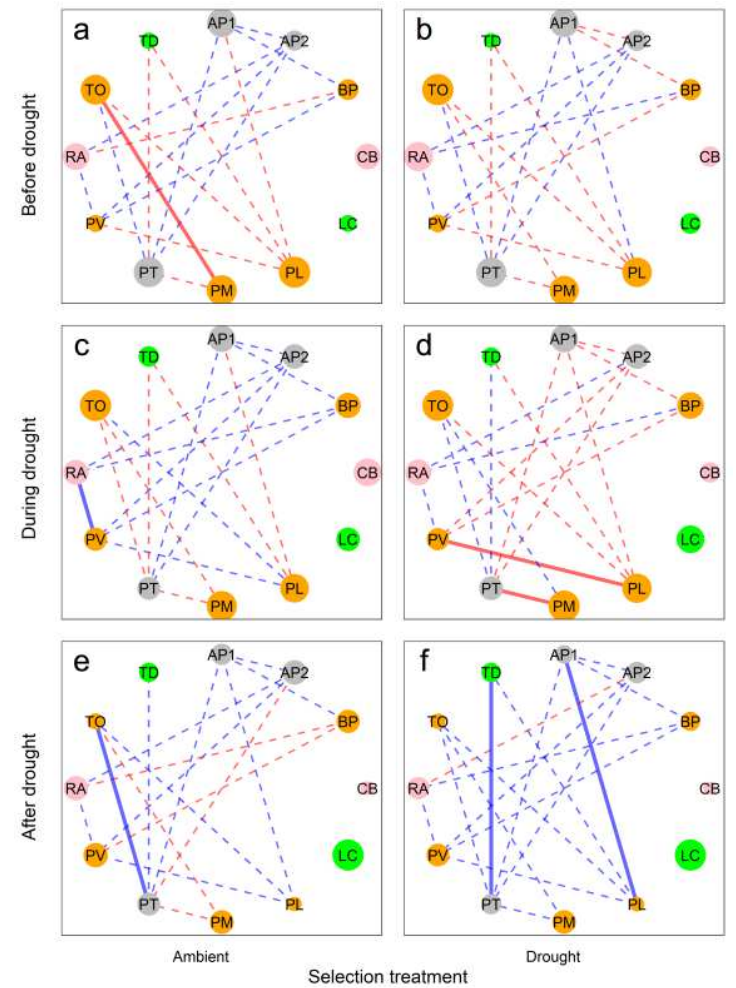

Figure 5 | Difference between heterospecific and conspecific interactions for species pairs of different selection treatments (ambient- vs. drought-selected plants) at each harvest (before $[a-b]$, during $[c-d]$ and after $[e-f]$ the drought event in the glasshouse). Lines connecting nodes (species) indicate species pairs within mixtures in the glasshouse. Blue lines indicate weaker heterospecific than conspecific competition, or stronger heterospecific than conspecific facilitation, while red lines represent the opposite situations. The solid lines indicate significant difference from zero based on Student's t test $(\alpha=0.05$; mixtures of each selection treatment with less than three replicates at a harvest were not tested for significance

871 and hence not shown in this figure). Node sizes are proportional to the corresponding monoculture biomass in each panel. Node colors indicate different functional groups

873 (green=legume, grey $=$ grass, orange $=$ short herb, pink=tall herb). Full species names are shown in Supplementary Table 1. 

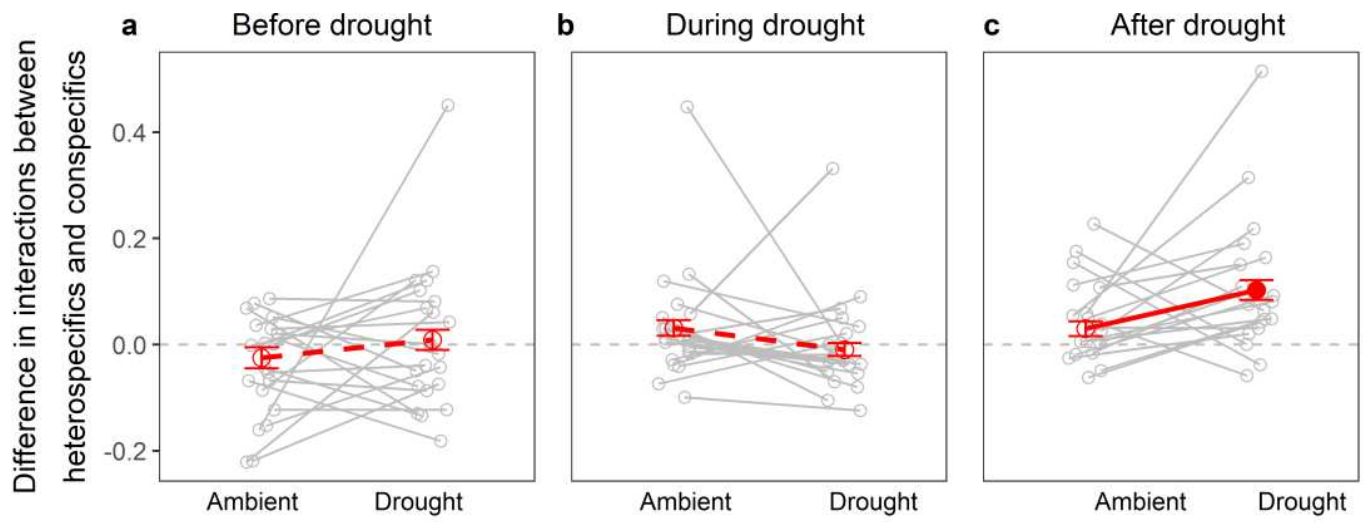

Figure 6 | Effects of selection treatment (ambient- vs. drought-selected plants) on

879 indicates a significant difference between the two selection treatments (see

880 Supplementary Table 8). Red points and error bars show means \pm standard error.

881 The filled red point shows a significant difference from zero (horizontal dashed lines; see Supplementary Table 7), i.e., weaker heterospecific than conspecific competition.

883 Grey points represent means for species pairs (standard errors for species pairs were not shown). Grey lines connect the same species pair between the two selection treatments. 


\section{Supplementary Files}

This is a list of supplementary files associated with this preprint. Click to download.

- DroughtexperimentSupplV9.pdf 